\title{
Quantum chaos in many-body systems: what can we learn from the Ce atom?
}

\author{
V.V. Flambaum, A.A. Gribakina, G.F. Gribakin *, I.V. Ponomarev \\ School of Physics, The University of New South Wales, Sydney 2052, Australia
}

\begin{abstract}
Results of an extensive study of a real quantum chaotic many-body system - the Ce atom - are presented. We discuss the origins of the quantum chaotic behaviour of the system, analyse statistical and dynamical properties of the multi-particle chaotic eigenstates and consider matrix elements or transition amplitudes between them. We show that based on the universal properties of the chaotic eigenstates a statistical theory of finite few-particle systems with strong interaction can be developed. We also discuss such important physical effects as enhancement of weak perturbations in many-body quantum chaotic systems, distribution of single-particle occupation numbers and its deviations from the standard Fermi-Dirac shape, and ways of introducing statistical temperature-based description in such systems. (C)1999 Elsevier Science B.V. All rights reserved.
\end{abstract}

PACS: 05.30.Fk; 05.45.+b; 24.60.Lz; 31.50.+w

Keywords: Quantum chaos; Finite Fermi systems

\section{Introduction}

The main purpose of the present work is to investigate the behaviour of conservative finite quantum systems of several strongly interacting particles. Examples of such systems are nuclei, atoms, molecules, atomic clusters or quantum dots in solids. Under certain conditions which we discuss below, the spectra of eigenvalues and the structure of the corresponding many-particle eigenstates acquire universal features. For example, the level spacing statistics become close to those of the Wigner random matrices, and the transition amplitudes between the eigenstates appear as uncorrelated Gaussian random variables.

\footnotetext{
*Corresponding author. E-mail: gribakin@newt.phys.unsw. edu.au.
}

The interactions between particles or degrees of freedom are very different in the many-body systems mentioned above. However, these systems have much in common. In the first approximation one chooses a particular mean field (e.g., that given by the Hartree-Fock method in atoms, or the adiabatic Born-Oppenheimer approximation in molecules) and uses it to construct a set of single-particle states. Many-particle states $\left|\Phi_{k}\right\rangle$ are then obtained by simply distributing the active particles among the singleparticle states. Such many-particle states are sometimes called configurations. Because of the residual (i.e., not included in the mean field) two-body interaction between the particles these many-particle states are not eigenstates of the system. However, they can be used as a many-particle basis set to construct the Hamiltonian matrix and find the eigenstates via its diagonalization. 
In the ground state of the system the particles just fill the lowest available single-particle states, hence, the ground state is usually characterized by a welldefined configuration. The admixture of higher lying configurations is rather small and can be taken into account by perturbations. As the energy of the system increases the number of single-particle states available for the active particles becomes large. For simple combinatorial reasons the number of many-particle states that can be constructed from them grows exponentially. The basis state energies, which can be defined as the expectation values of the Hamiltonian, form a very dense mesh and the mean interval between neighbouring basis state energies becomes very small. Under these conditions even a small residual interaction introduces strong nonperturbative mixing of the basis states. Roughly speaking, this happens when the configuration-mixing off-diagonal matrix elements $H_{i j}$ of the Hamiltonian become greater than the energy spacing between the basis states coupled by the residual interaction (see, e.g., [1-4]).

As a result, the following structure of the eigenstates is established. Each eigenstate is a linear combination of the basis states, $|\Psi\rangle=\sum_{k} C_{k}\left|\Phi_{k}\right\rangle$. The number of basis states that strongly participate in a given eigenstate is large, $N \gg 1$. It can be estimated as $N \sim \Gamma / D$, where $\Gamma \simeq 2 \pi \overline{H_{i j}^{2}} \rho$ is the so-called spreading width, $\rho=D^{-1}$ is the eigenvalue density, and $D$ is the mean level spacing between the eigenvalues (approximately equal to the mean spacing between the basis state energies). The value of $\Gamma$ is usually comparable with typical energy scales in the problem, thus, $\Gamma \sim \mathrm{eV}$ in atoms and $\Gamma \sim \mathrm{MeV}$ in nuclei. For basis states $k$ whose energies are close to the eigenvalue, $\left|E_{k}-E\right| \lesssim \Gamma$, the components are large $C_{k} \sim 1 / \sqrt{N}$, and these components dominate the normalization condition $\sum_{k} C_{k}^{2}=1$. Outside the spreading width, at $\left|E_{k}-E\right|>\Gamma$, the components decrease. These regular features aside, the components behave like random variables. In other words, the meansquared value of the component $\left\langle C_{k}^{2}\right\rangle$ is a smooth function of $E_{k}-E$ with a maximum at $E_{k} \approx E$. For fixed $E_{k}-E$ the statistics of $C_{k}$ is close to Gaussian.
In this situation the eigenvalue spectrum is characterized by level repulsion effects and the statistics of level spacings is described by the famous Wigner formula. Note that in common random matrices, e.g., those of the Gaussian orthogonal ensemble, the diagonal matrix elements fluctuate similarly to the offdiagonal ones, whereas the diagonal matrix elements of the many-body Hamiltonian increase monotonically. ${ }^{1}$ Studies of experimental data for the energy levels in heavy nuclei [5] and complex atoms [6,7] agree with the Wigner statistics. They have been observed in numerical calculations for the atom of cerium (Ce) [8] and the nuclear sd shell model [9-12].

When every eigenstate is a chaotic superposition of a large number of basis states the eigenstates loose their "individual features", and the only good quantum numbers remaining in the spectrum are the exact ones: the total angular momentum and parity (if the Hamiltonian is symmetric with respect to rotation and inversion), and the energy itself. Since different configurations are mixed together by the residual interaction, the occupation numbers of the single-particle orbitals strongly deviate from integers and the eigenstates cannot be characterized in terms of the singleparticle quantum numbers. The matrix elements of some external perturbation (transition amplitudes) between the chaotic eigenstates have the statistics of a random Gaussian variable with zero mean. ${ }^{2}$ Note that all these effects take place in the energy range of pure quantum dynamics, well below the semiclassical limit. However, the picture of chaotic "compound" (nuclear physics term) eigenstates produced by the strong residual interaction between the particles allows one to describe this situation as many-body quantum chaos.

\footnotetext{
${ }^{1}$ Of course, this can always be achieved by appropriate enumeration of the basis states. The physical importance of the diagonal matrix elements is in that they essentially guide the behaviour of the eigenvalue density in the system. On the contrary, in many random matrix models the distribution of the diagonal matrix elements is narrow, e.g., Gaussian or rectangular, and the eigenvalues spread over a much wider energy range. The eigenvalue density is then determined by the mean-squared off-diagonal matrix element and has the shape of semicircle.

2 Their distribution can therefore be characterized by the variance, or the mean-squared value, alone. Its value varies smoothly as a function of the energies of the eigenstates involved (secular variation).
} 
The properties of the chaotic eigenstates can be used to develop a statistical theory of finite few-particle quantum systems [13-15]. The specific equilibrium that emerges in the system due to the residual twobody interaction enables one to introduce thermodynamic temperature-based description in the isolated few-body system $[9,16]$ and use it, e.g., to calculate average occupation numbers of the single-particle states. This is possible in spite of large deviations of the occupation numbers from the usual Fermi-Dirac distribution caused by the strong interaction between particles [16]. Based on the structure of the many-body compound states a statistical approach to calculation of matrix elements (transition amplitudes) between these states has been developed $[17,18]$. It expresses the mean-squared matrix elements of an operator in terms of the single-particle amplitudes and occupation numbers, and characteristics of the chaotic eigenstates involved, namely, their energies, spreading widths and numbers of principal components $N$.

One of the most interesting properties of the chaotic many-body systems is dynamical enhancement of perturbations. It is responsible for the huge $10^{6}$-times enhancement of the weak interaction in compound nuclei (see, e.g., $[19,20])$. Due to this phenomenon parity nonconservation effects at $10 \%$ level have been observed in neutron scattering by heavy nuclei. The origin of the enhancement is in that the typical level spacing in the chaotic many-body system is very small, $D \sim \Gamma / N$, where $N$ can be as large as $10^{6}$ in nuclei. The matrix element of a perturbation between the chaotic many-body eigenstates is also suppressed, but only as $1 / \sqrt{N}$. Therefore, the effect of the perturbation, estimated as the ratio of its matrix element to the energy denominator, is $\sqrt{N}$ times enhanced. Note that the strong mixing of the basis states by the residual interaction is essential for the dynamical enhancement. It cannot be observed in a system of noninteracting particles, e.g., a perfect gas, although its energy spectrum can be very dense. The point is that nearby multi-particle levels in this case will have very different configurations and will not be coupled by a one-or two-body external perturbation.

The purpose of this paper is three-fold. Using our numerical calculations of the real four-particle system, the $\mathrm{Ce}$ atom, we illustrate the properties of chaotic many-body eigenstates and show how they can be used to develop a statistical theory for such systems. Secondly, we analyse to what extent our realistic numerical model agrees with usual assumptions and conclusions of the random-matrix theories (see reviews $[21,22])$. Thirdly, we demonstrate that complex openshell atoms are convenient testing grounds for studying many-body quantum chaos, open for both theoretical and experimental investigation.

It looks appropriate to give a brief overview of the problem of quantum chaos in atoms. Since Bohr's theory of the hydrogen atom atoms were considered as perfectly regular dynamical quantum systems. However, as the theory of classical chaos evolved, it became clear that highly excited atomic states in the Rydberg range could become chaotic if an external field is applied [23,24], as long as the underlying classical motion was chaotic.

On the other hand, it was also due to Bohr that the notion of compound nuclei was introduced in physics. The behaviour of these highly excited nuclear states is essentially quantum-mechanical. Nevertheless, they display a number of chaotic properties described above. The first insight into quantum chaotic properties of complex atoms was given by Rosenzweig and Porter [6] who analysed experimental spectra of some neutral atoms and showed that the spectral statistics of heavy open-shell atoms are similar to those of compound nuclei. That analysis was later extended and refined in [7]. Of course, the study of eigenvalues provides valuable information about the system. On the other hand, the spectral statistics observed in the heavy open-shell atoms are similar to those of the hydrogen atom in a strong magnetic field [25], or even a particle in a two-dimensional classically ergodic billiard [26,27]. However, the eigenstates of these quantum systems must be completely different, and it is clear that the eigenvalue statistics cannot really tell us much about the origin of chaotic behaviour, or indeed the structure of the chaotic eigenstates.

The first inquiry into the possibility of chaos in the eigenstates of complex atoms was done by Chirikov [28]. He studied configuration compositions of the eigenstates of the $\mathrm{Ce}$ atom using data from the tables 
[29], and came to the conclusion that the "eigenfunctions are random superpositions of some few basic states". Inspired by that work we conducted an extensive numerical study of the spectra and eigenstates of complex open-shell atoms, using the rare-earth atom of Ce as an example [8,16,30-32]. The present paper summarizes our earlier findings, as well as gives new insights into the problem of quantum chaos in a real many-body system.

\section{Eigenvalues and eigenstates}

\subsection{The cerium atom}

The atom of $\mathrm{Ce}(Z=58)$ has one of the most complicated spectra in the periodic table. Besides their energies, the atomic eigenstates are characterized by the total angular momentum $J$ and parity $\pi$ ( + or $-)$. For a given $J^{\pi}$ the level density in Ce reaches hundreds of levels per $\mathrm{eV}$ at excitation energies of just few eV, well below the ionization threshold of $I=$ $5.539 \mathrm{eV}$ [29]. The electronic structure of Ce consists of a Xe-like $1 \mathrm{~s}^{2} \ldots 5 \mathrm{p}^{6}$ spherically symmetric core and four valence electrons - active particles ${ }^{3}$. The atomic ground state is described by the $4 \mathrm{f} 6 \mathrm{~s}^{2} 5 \mathrm{~d}$ configuration with $J^{\pi}=4^{-}$.

The origin of extremely complex and dense excitation spectra in $\mathrm{Ce}$ and other rare-earth atoms is in the existence of several open orbitals $n l j$ near the ground state, namely, $4 \mathrm{f}_{5 / 2}, 4 \mathrm{f}_{7 / 2}, 6 \mathrm{~s}_{1 / 2}, 5 \mathrm{~d}_{3 / 2}, 5 \mathrm{~d}_{5 / 2}, 6 \mathrm{p}_{1 / 2}$, and $6 \mathrm{p}_{3 / 2}$. Each of the orbitals is $2 j+1$-degenerate and this makes a total of $N_{\mathrm{s}}=32$ single-electron states. For Ce with $n=4$ valence electrons there are about $\left(N_{\mathrm{s}}\right)^{n} / n ! \approx 4 \times 10^{4}$ possible many-electron states constructed from them. If we allow for the two possible parities, about ten possible values of $J$, and $2 J+1$ angular momentum projections $J_{z}$ (another factor of 10), there will still be hundreds of energy levels within a given $J^{\pi}$ manifold.

\footnotetext{
${ }^{3}$ The typical excitation energy of the core is about $20 \mathrm{eV}$. Below this energy we can work in the "frozen core" approximation and consider the wave function of the core as a "vacuum" state $|0\rangle$, to which the four valence electrons are added.
}

\subsection{Energy levels}

The calculations are performed using the HartreeFock-Dirac (HFD) and configuration interaction methods. A self-consistent HFD procedure determines the mean-field potential of the atom and calculates the basis set of single-particle states for the active (valence) electrons, $|\alpha\rangle=\left|n l j j_{z}\right\rangle$ with energies $\varepsilon_{\alpha}$. This procedure defines the zeroth-order Hamiltonian of the system,

$\hat{H}^{(0)}=\sum_{\alpha} \varepsilon_{\alpha} a_{\alpha}^{\dagger} a_{\alpha}$.

The multi-particle configuration basis states (determinants) $\left|\Phi_{k}\right\rangle$ are constructed from the single-particle states, $\left|\Phi_{k}\right\rangle=a_{v_{1}}^{\dagger} a_{v_{2}}^{\dagger} a_{v_{3}}^{\dagger} a_{\nu_{4}}^{\dagger}|0\rangle$. By construction $\left|\Phi_{k}\right\rangle$ are eigenstates of the $\hat{J}_{z}$ operator. To account for the conservation of the total angular momentum in the system, a new symmetrized basis $\left|\Phi_{k}^{(J)}\right\rangle, \hat{J}^{2}\left|\Phi_{k}^{(J)}\right\rangle=$ $J(J+1)\left|\Phi_{k}^{(J)}\right\rangle$, is obtained by a linear transformation of $\left|\Phi_{k}\right\rangle$.

The total Hamiltonian $\hat{H}$ of the active electrons is the sum of the mean-field Hamiltonian of the core $\hat{H}^{(0)}$ and the two-body residual interaction:

$\hat{V}=\frac{1}{2} \sum_{\alpha \beta \gamma \delta} V_{\alpha \beta \gamma \delta} a_{\alpha}^{\dagger} a_{\beta}^{\dagger} a_{\gamma} a_{\delta}$,

where $V_{\alpha \beta \gamma \delta}$ is the matrix element of the Coulomb interaction between the electrons. In the $\left|\Phi_{k}^{(J)}\right\rangle$ basis the Hamiltonian matrix has a block diagonal structure, $\left\langle\Phi_{i}^{(J)}|\hat{H}| \Phi_{k}^{\left(J^{\prime}\right)}\right\rangle=H_{i k}^{(J)} \delta_{J J^{\prime}}$. Since we always consider states with a given $J$ (and parity), the corresponding superscript will be dropped hereafter.

The diagonal matrix elements $H_{k k}=H_{k k}^{(0)}+V_{k k} \equiv$ $E_{k}$ can be interpreted as energies of the basis states. The off-diagonal matrix elements $H_{i k}=V_{i k}$ are responsible for mixing of the multi-particle basis states. Diagonalization of the Hamiltonian matrix yields the energies $E^{(i)}$ and the eigenstates $\left|\Psi_{i}\right\rangle=\sum_{k} C_{k}^{(i)}\left|\Phi_{k}\right\rangle$ of the system.

In our earlier work [8] we considered only a few lowest nonrelativistic configurations constructed of the 4f, 6s, 5d, and 6p orbitals, and had 260 and 276 eigenstates of the $J^{\pi}=4^{-}$and $4^{+}$symmetries, respec- 


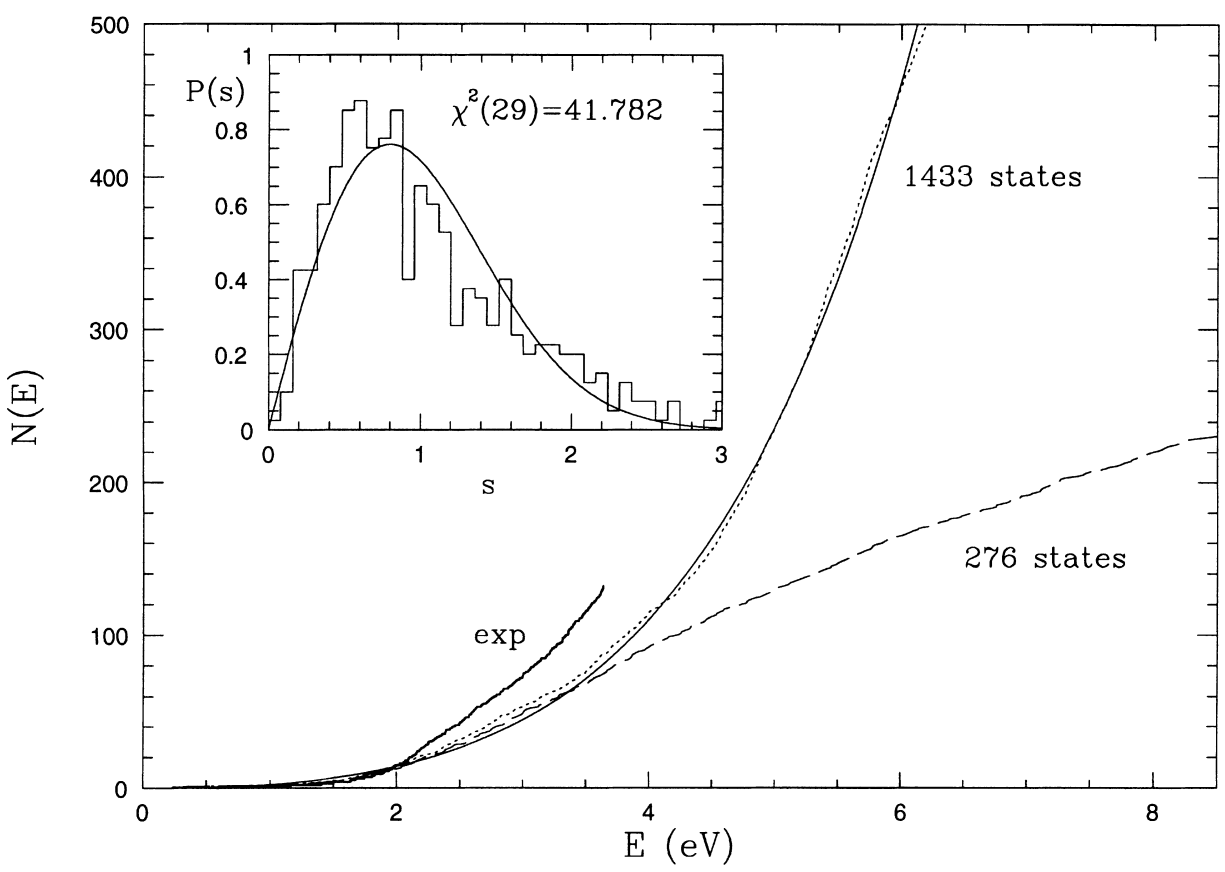

Fig. 1. Cumulative number of levels and level spacing statistics for the $J^{\pi}=4^{+}$states in Ce. Dotted line is the calculation with 1433 basis states; dashed line is the small-scale calculation with 276 states; thick solid line describes 132 experimental levels from [29]. Thin solid line is the independent-particle fit (4). The inset shows statistics of the normalized level spacings $s$ for the 500 levels, compared with the Wigner distribution (5).

tively. To make the results more realistic we have increased the single-particle basis by including the $5 \mathrm{f}$, $7 \mathrm{~s}, 7 \mathrm{p}$ and $6 \mathrm{~d}$ orbitals, and extended the configuration basis set by including all electron configurations within $10 \mathrm{eV}$ of the atomic ground state. This increased the total number of $4^{-}$and $4^{+}$states to 862 and 1433 , respectively ${ }^{4}$.

Fig. 1 shows the calculated cumulative number of levels

$N(E)=\int_{-\infty}^{E} \rho\left(E^{\prime}\right) \mathrm{d} E^{\prime}$

where $\rho(E)=\sum_{i} \delta\left(E-E^{(i)}\right)$ is the level density, for the $J^{\pi}=4^{+}$eigenstates of Ce. Note that the energy scale is chosen so that the $E=0$ corresponds to the energy of the $\mathrm{Ce} 4^{-}$ground state. The main feature

\footnotetext{
${ }^{4}$ For the given choice of the basis the numbers of positive-parity eigenstates with $J=0-10$ were $343,917,1354,1493,1433,1153$, $826,497,262,107$, and 34 , respectively, i.e., the full size of our Hilbert space is about $8 \times 10^{3}$. We have chosen to analyse the states with $J=4$, as this manifold is among the most abundant.
}

of the spectrum is a rapid growth of the level density with energy (cf. our earlier small-scale calculation). In the independent-particle model this dependence is described by the following exponent [5]:

$\rho_{a}(E)=\rho_{0} \exp \left[a\left(E-E_{\mathrm{g}}\right)^{1 / 2}\right]$

where $E_{\mathrm{g}}$ is the ground state energy. It can be seen from Fig. 1 that for $\rho_{0}=0.65 \mathrm{eV}^{-1}, a=2.55 \mathrm{eV}^{-1 / 2}$ and $E_{\mathrm{g}}=0.25 \mathrm{eV}$ (energy of the lowest $4^{+}$level with respect to the $4^{-}$Ce ground state), Eq. (4) gives a good overall fit of the level density. The experimental spectra of the rare-earth atoms and their ions examined in [7] are also in agreement with Eq. (4).

The second feature typical for the spectra of complex many-body systems is level repulsion. It is described well by the random-matrix theory. A good approximation to the distribution of level spacings is given by the Wigner formula

$P(s)=(\pi s / 2) \exp \left(-\pi s^{2} / 4\right)$, 
where $s$ is the nearest-neighbour level spacing normalized as $\bar{s}=\int s P(s) \mathrm{d} s=1$. As we pointed out in Section 1, spectral statistics do not tell much about the eigenstates of the system. However, Eq. (5) is still a good test for some possible hidden quantum numbers, e.g., the total spin or orbital momentum, which might characterize atomic eigenstates besides $J^{\pi}$. If those did exist small level spacings ("degeneracies") would be more abundant than that predicted by Eq. (5). The spectral statistics were checked for many experimental and calculated complex atomic spectra [6-8,33-35], as well as for molecular vibronic spectra [36].

On the inset in Fig. 1 we compare Eq. (5) with the level spacing distribution for the 500 lowest $4^{+}$ states in Ce. The calculated level spacings were normalized using the analytical density fit: $s_{n}=\left(E_{n+1}-\right.$ $\left.E_{n}\right) \rho_{a}\left(E_{n}\right)$. The agreement is good and the deviations are probably due to the long-range fluctuations of the level density, not accounted for by the simple exponential (4).

Thus, we see that the eigenvalue density in the many-body system indeed rapidly increases with energy. The level spacing distribution evidences that there is strong nonperturbative configuration mixing in the system. The energy, total angular momentum and parity remain the only good quantum numbers.

\subsection{Chaotic eigenstates}

Fig. 2(a) depicts a typical eigenstate of Ce. It shows the components $C_{k}$ of the 400th $4^{+}$eigenstate vs. the energies of the basis states $E_{k}$. The energy of the 400th even state is $E=5.73 \mathrm{eV}$ above the Ce ground state. The contributions of the basis states whose energies are close to the eigenvalue are large, whereas for $E_{k}$ further away from the eigenvalue the components show a steady decrease. In general the large (principal) components are centred around some energy $E+\Delta E$ slightly shifted away from the eigenvalue. This effect is especially strong near the edges of the spectrum. Thus, at the low energy end of the spectrum the eigenvalues are systematically shifted down with respect to the diagonal matrix elements of the Hamiltonian. This is just another manifestation of the level repulsion due to the off-diagonal matrix elements.

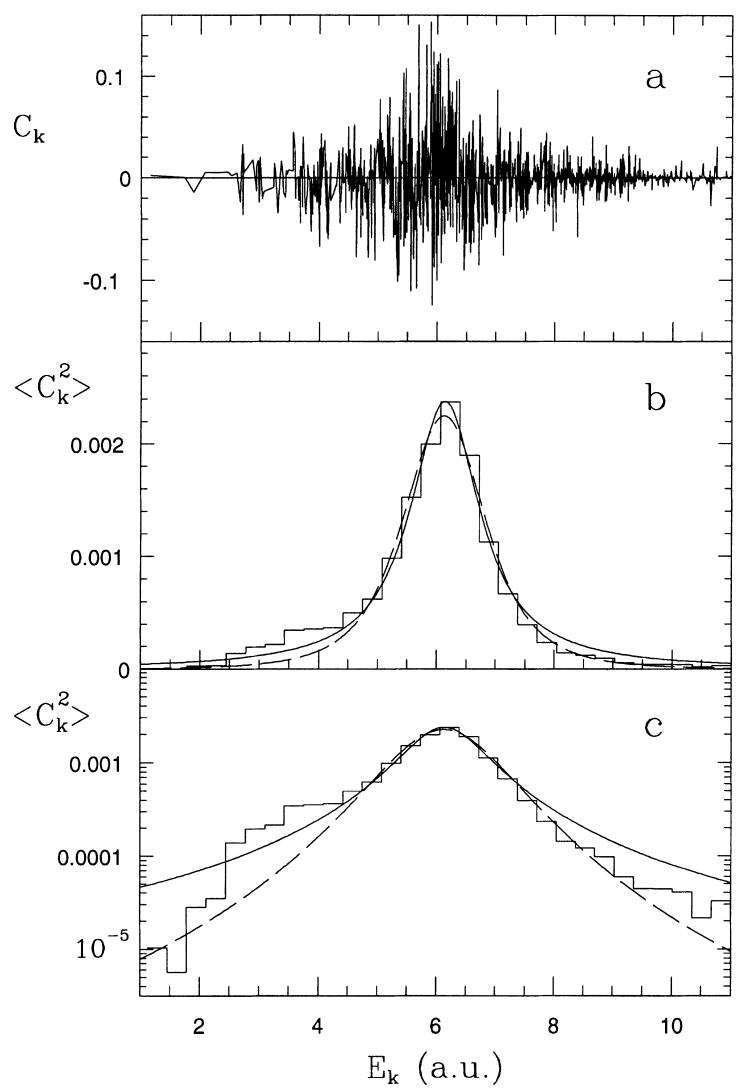

Fig. 2. Components of the 400th $J^{\pi}=4^{+}$eigenstate (a) and the mean-squared values for the $400 \pm 9$ eigenstates (histogram) (b) fitted by a Breit-Wigner (solid curve, Eq. (7), $N=421$, $\Gamma=1.44 \mathrm{eV}$ ) and squared Breit-Wigner (dashed curve) functions. Plot (c) is done using a logarithmic scale to magnify $\left\langle C_{k}^{2}\right\rangle$ at the wings, for $\left|E_{k}-E\right|>\Gamma$.

Therefore, the many-body eigenstates appear to be chaotic and localized at $E_{k} \approx E$ with respect to the configuration-state basis $\left|\Phi_{k}\right\rangle$. The latter property is best characterized by the strength function introduced by Wigner and also known as the local density of states:

$\rho_{\mathrm{W}}(E, k)=\sum_{i} C_{k}^{(i) 2} \delta\left(E-E^{(i)}\right) \simeq\left\langle C_{k}^{2}\right\rangle_{E} \rho(E)$,

where $\left\langle C_{k}^{2}\right\rangle_{E} \equiv\left\langle C_{k}^{(i) 2}\right\rangle$ is the mean-squared component averaged over the eigenstates with $E^{(i)} \approx E$, and $\rho(E)$ is the eigenvalue density. We present $\left\langle C_{k}^{2}\right\rangle_{E}$ obtained by averaging the components of the $400 \pm 9$ th eigenstates within discrete energy intervals in Fig. 2(b). Its shape is described well by the Breit-Wigner 
(BW) profile

$$
\left\langle C_{k}^{2}\right\rangle_{E}=\frac{1}{N} \frac{\Gamma^{2} / 4}{\left(E_{k}-E-\Delta E\right)^{2}+\Gamma^{2} / 4},
$$

which also defines the spreading width $\Gamma$ and the number of principal components $N$. Since $\left\langle C_{k}^{2}\right\rangle=1 / N$ at the centre of the eigenstates, the value of $N$ characterizes the number of eigenstate components around the maximum of the profile. By means of the normalization $\sum_{k}\left\langle C_{k}^{2}\right\rangle_{E}=\sum_{k} C_{k}^{(i) 2}=1$, Eq. (7) implies $N=$ $\pi \Gamma / 2 D$, where $D=1 / \rho$ is the mean level spacing.

The BW shape of the strength function was first derived for the infinite-size band random matrix (BRM) model $[37,38]$. It was assumed in the model that the diagonal matrix elements are equally spaced $H_{k k}=k D$ (thus, $\rho=D^{-1}=$ const), and the off-diagonal matrix elements are independent random variables with $\overline{H_{i j}}=0$, and $\overline{H_{i j}^{2}}=V^{2}$ for $|i-j| \leq b(b \gg 1$ characterizes the width of the band) and $H_{i j}=0$ outside the band. Wigner showed that inside the band, for $\left|E_{k}-E\right|<D b$, the strength function is given by Eq. (7) with $\Gamma=2 \pi V^{2} \rho$, provided the eigenstates are localized within the band: $\Gamma<D b$. Recently this result has been derived for sparse matrices with a diffuse band [39]. The BW spreading also emerges in the well-known nuclear physics model of a state interacting with a large set of states with $\rho=$ const by means of a constant or weakly fluctuating matrix element [5]. It has been verified numerically in our earlier calculations in Ce [8], the sd shell nuclear model [9-12] (for the interaction strengths which satisfied $\Gamma<D b$ ), and the two-body random interaction model [40]. Some of the deviations from the BW shape observed in [9-12] were attributed to the fact the $\rho \neq$ const. in realistic calculations. They also noticed that $\left\langle C_{k}^{2}\right\rangle_{E}$ demonstrates a much better agreement with the $\mathrm{BW}$ profile than the strength function (6) itself.

If we use the parameters of the BW fit we can estimate $D=\pi \Gamma / 2 N \approx 5.4 \times 10^{-3} \mathrm{eV}, \rho=185 \mathrm{eV}^{-1}$ near the 400th even eigenstate (a similar number is obtained from Eq. (4)). Combined with the mean-squared off-diagonal matrix element for the $J^{\pi}=4^{+}$states, $\overline{H_{i j}^{2}}=1.22 \times 10^{-3} \mathrm{eV}^{2}$ (see Section 2.4) we can obtain a theoretical value of $\Gamma=2 \pi \overline{H_{i j}^{2}} \rho=1.42 \mathrm{eV}$, in agreement with that obtained from the BW fit in Fig. 2(b). There are two reasons for discrepancies between the numerical $\left\langle C_{k}^{2}\right\rangle_{E}$ and the BW shape. Firstly, there are some configurations which mix better than others, i.e., $\overline{H_{i j}^{2}}$ for them is greater than that for the whole matrix. Hence, one observes a shoulder-like structure on the low-energy side of the $\left\langle C_{k}^{2}\right\rangle_{E}$ maximum. Secondly (see Fig. 2(c)), as $\left|E-E_{k}\right|$ increases, the squared components tend to drop faster than that predicted by Eq. (7). This effect is emphasized by the squared Breit-Wigner fit in the figure. It is caused by an effective bandedness of the Hamiltonian matrix, which means that for greater $\left|E-E_{k}\right|$ the coupling decreases, and the mixing is achieved effectively through higher perturbation theory orders. This becomes especially obvious in the Wigner BRM model where the decrease of the strength function outside the band is exponential $[8,37,38]$.

The systematic behaviour of the components' variance is described by $\left\langle C_{k}^{2}\right\rangle_{E}$. Apart from this their statistics at a given $E_{k}-E$ should become Gaussian with zero mean if the basis state mixing is complete and uniform. We observed this effect earlier by analysing the statistics of the normalized components $C_{k}^{(i)}\left[\left\langle C_{k}^{2}\right\rangle_{E}\right]^{-1 / 2}$ [8]. If on the contrary there were subsystems not coupled or coupled weakly by the residual interaction, there would be a large abundance of zero or very small components. In the present calculation besides the lowest electron orbitals of each symmetry, $4 \mathrm{f}, 6 \mathrm{~s}, 5 \mathrm{~d}$ and $6 \mathrm{p}$ we had in [8], we have included orbitals with higher principal quantum numbers. They have larger radii and the residual Coulomb interaction is smaller for them. Nevertheless, Fig. 3 shows that the distribution of the normalized components of the 400th eigenstate used as an example is rather close to Gaussian.

The picture of chaotic many-body eigenstates outlined above is valid when the number of principal components $N$ is large. This happens when the excitation energy is much greater than the single-particle level spacing $d_{0}$. The effective value of $d_{0}$ can be estimated from the fitting parameter $a$ in Eq. (4) and its analytical value in the Fermi gas model [5], $a=$ $2\left[\pi^{2} g_{0} / 6\right]^{1 / 2}$, where $g_{0}$ is the single-particle level density at the Fermi level. Using $a=2.55 \mathrm{eV}^{1 / 2}$ we ob- 


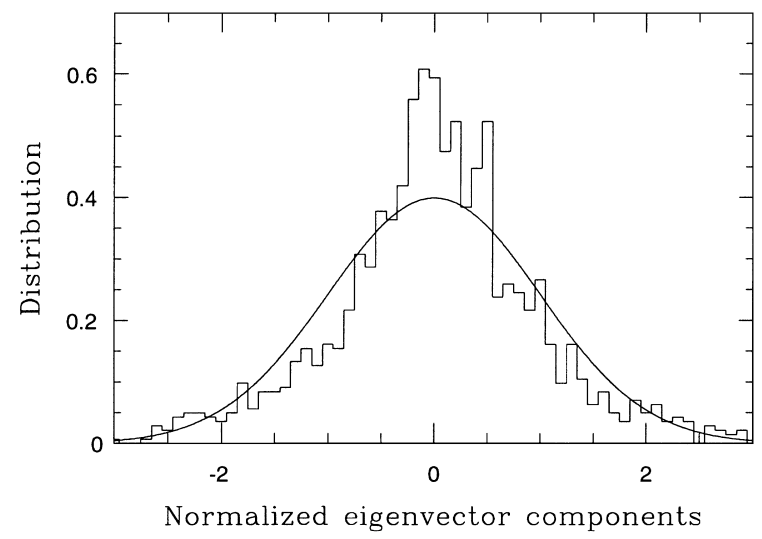

Fig. 3. Statistics of the normalized components $C_{k}^{(i)}\left[\left\langle C_{k}^{2}\right\rangle_{E}\right]^{-1 / 2}$ for the 400th $J^{\pi}=4^{+}$eigenstate (histogram), compared to the normal Gaussian distribution (solid curve).

tain $d_{0}=g_{0}^{-1}=1 \mathrm{eV}$. Practically, at a twice greater excitation energy $E-E_{\mathrm{g}}=2 \mathrm{eV}$, where $\rho \approx 25 \mathrm{eV}^{-1}$, the number of prinicipal components $N \sim \rho \Gamma \sim 40$ is already large.

We can compare this estimate with the condition for the onset of chaos presented in [1-4]. At this energy the spacing between the many-body states is $D \approx 0.04 \mathrm{eV}$. The spacing $d_{2}$ between the basis states coupled directly by $H_{i j}$ is approximately two times greater, $d_{2} \approx 0.08 \mathrm{eV}$, since the sparsity of the Hamiltonian matrix is $S \approx 0.5$ (see Section 2.4). The typical nonzero off-diagonal matrix element is $H_{i j} \sim$ $0.1 \mathrm{eV}$ (see below), thus, the condition of chaotic mixing $H_{i j} \gtrsim d_{2}$ is fulfilled.

\subsection{Statistics of the Hamiltonian matrix}

We have seen that the properties of the eigenvalues and eigenvectors of a real chaotic many-body system (Ce) are similar to those obtained in random matrix models. Of course, the Hamiltonian of $\mathrm{Ce}$ is in no sense random: this is a purely dynamic system driven only by the Coulomb interaction between the electrons and with the nucleus. Moreover, it is easy to check that the statistics of the off-diagonal Hamiltonian matrix elements shown in Fig. 4 bears very little resemblance to the Gaussian distribution adopted in many random-matrix studies. It should rather be described

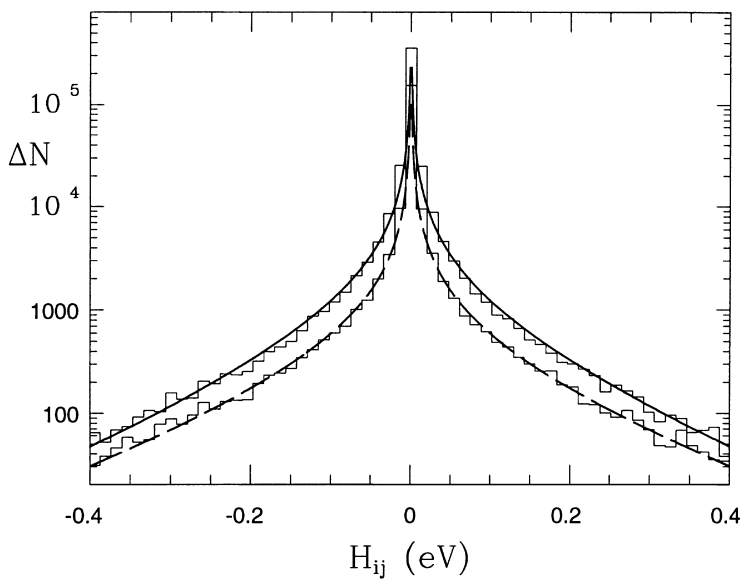

Fig. 4. Distribution of the off-diagonal matrix elements of the $J^{\pi}=4^{+} 1433 \times 1433$ (upper histogram) and $J^{\pi}=4^{-} 862 \times 862$ (lower histogram) Hamiltonian matrices. Thick solid and dashed curves show simple fits (8) with $\kappa=1$ and $V_{\kappa}=0.16$ and $0.19 \mathrm{eV}$, respectively.

by a singular expression

$P\left(H_{i j}\right) \propto\left|H_{i j}\right|^{-\kappa} \exp \left(-\left|H_{i j}\right| / V_{\kappa}\right)$,

where $\kappa>0$ and $V_{\kappa}$ characterizes the typical value of the matrix element.

In our previous work [8] we analysed the distribution of $H_{i j}$ for small Hamiltonian matrices in the configuration space built from the seven lowest electron orbitals, and adopted $k=1 / 2$. We see from Fig. 4 that addition of new configurations involving higherlying electron orbitals has increased the $\kappa$ value to 1 . This means that there is a larger fraction of small matrix elements in the Hamiltonian now. In the nuclear sd shell model values of $k=1$ and 2 were obtained, depending on the total angular momentum and isospin of the states. Note that for $\kappa \geq 1$ distribution (8) has an infinite norm. On the other hand the true dimension of the Hilbert space and the Hamiltonian matrix of a real many-body system is infinite. Accordingly, most of the matrix elements describe mixing between very distant and different configurations, hence, they must be very small. The fact that we obtain $\kappa \geq 1$ in realistic numerical calculations is probably a manifestation of this general phenomenon.

Fig. 5 gives a better insight into the distribution of the matrix elements $H_{i j}$ over the matrix. We have not 

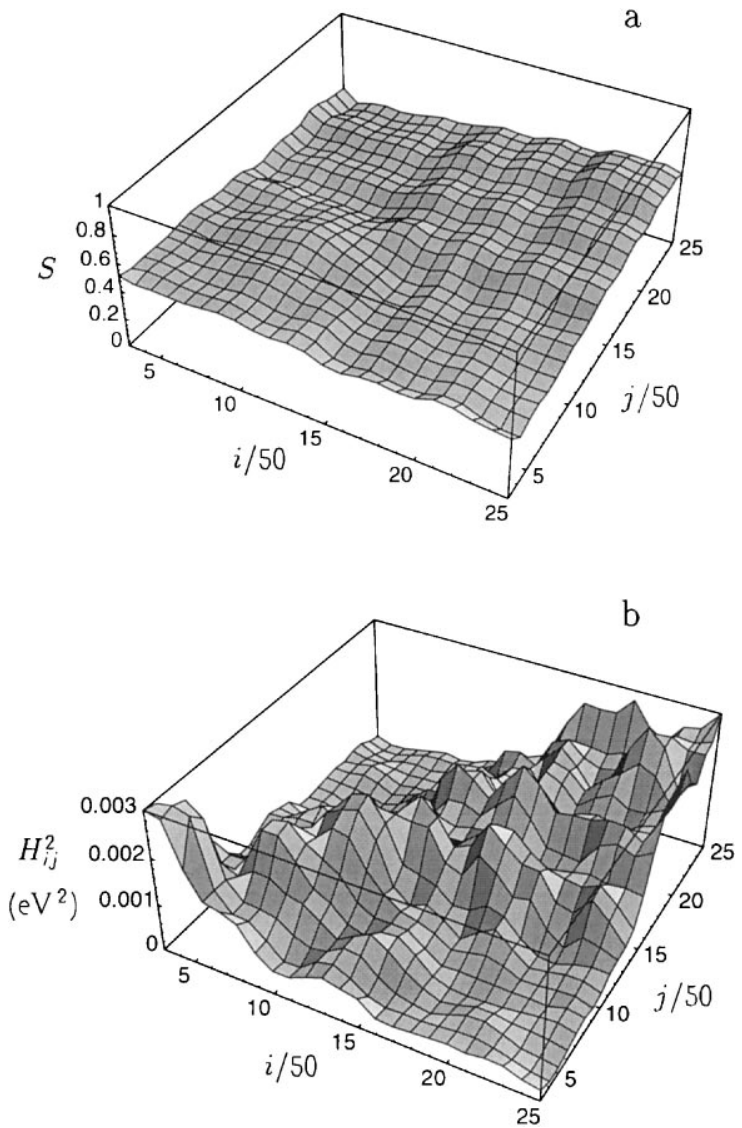

Fig. 5. Properties of the Hamiltonian matrix of the $J^{\pi}=4^{+}$ states obtained by averaging over a running $99 \times 99$ window: (a) sparsity; (b) mean-squared matrix elements.

mentioned yet that the Hamiltonian matrix of a manybody system with two-body interaction between particles is always sparse if the number of active particles is greater than 2 . In this case there are basis states $\left|\Phi_{i}\right\rangle$ and $\left|\Phi_{j}\right\rangle$ that differ by positions of more than two particles, which means that the two-body residual interaction (2) does not couple them: $H_{i j}=\left\langle\Phi_{i}|\hat{V}| \Phi_{j}\right\rangle=0$. Thus, the Hamiltonian matrix contains a certain number of zero off-diagonal elements. This property is better characterized by specifying the fraction $S$ of nonzero matrix elements, which we call sparsity. If all $H_{i j}$ are nonzero, $S=1$. The Hamiltonian matrix calculated in the determinant basis is quite sparse $(S \ll 1)$, because the number of single-particle states is usually much greater than the number of active particles (32 and 4 in our original calculation of $\mathrm{Ce}$, or 24 and
12 in [9-12]). In the symmetrized $J^{\pi}$ basis there are much fewer zero matrix elements $H_{i j}$ due to a certain "pre-mixing" of the determinants through the angularmomentum algebra ${ }^{5}$. As a result, just over $50 \%$ of $H_{i j}$ are zeros in our calculation of the $J^{\pi}=4^{+}$states of Ce. As seen in Fig. 5(a) $S$ is almost constant over the matrix. The average sparsity for the whole matrix is $S=0.45$.

On the contrary, the locally averaged squared matrix elements $\overline{H_{i j}^{2}}$ show a considerable variation, Fig. 5(b). In spite of the roughness of the $\overline{H_{i j}^{2}}$ surface one can clearly see the existence of a wide and diffuse band with $b \sim 500$. Inside this band the matrix elements are noticeably larger than the mean-square offdiagonal matrix element for the whole matrix $\overline{H_{i j}^{2}}=$ $1.22 \times 10^{-3} \mathrm{eV}^{2}$. Note that the rms value calculated over the nonzero $H_{i j}(0.052 \mathrm{eV})$ is of the same order of magnitude as the $V_{\kappa}$ parameter in Fig. 4. The existence of the band in the Hamiltonian matrix explains the faster-than-BW decrease of the mean-squared components at the wings $\left|E_{k}-E\right| \gtrsim D b$ seen in Fig. 2(c).

The banded structure of the Hamiltonian matrix was also seen in our early calculations for Ce [30] and in the nuclear sd shell model [9-12]. Thus, it appears that the Hamiltonian matrix of a real chaotic many-body system can be described as both sparse and banded, with a singular distribution (8) of the nonzero matrix elements. However, the parameters of the matrix, like the mean spacing between the diagonal matrix element, the mean-squared off-diagonal matrix element and the effective width of the band may vary along the matrix.

\section{Occupation numbers in the few-body Fermi system}

\subsection{Equilibrium brought by the interaction between particles}

The picture of chaotic mixing of basis states revealed in Section 2 means that the strong residual

\footnotetext{
${ }^{5}$ This effect has been described as "geometrical chaoticity" in [9-12].
} 
two-body interaction between the active particles introduces some kind of statistical equilibrium in the system. It is governed by the large number of components $N$ mixed together within every eigenstate of the system ${ }^{6}$. Accordingly, the size of fluctuations is $\sim 1 / \sqrt{N}$. This situation is different from the standard statistical mechanical notion of the equilibrium that requires the number of particles or degrees of freedom in the system to be large. On the contrary, the equilibrium in $\mathrm{Ce}$ is achieved with just four electrons.

This equilibrium enables one to develop a statistical theory for finite few-particle quantum systems with strong interaction between the particles $[14,15]$. This theory should allow one to introduce temperature, entropy, etc., and calculate various properties of the system, e.g., the occupation numbers, or the rms values of matrix elements of an external perturbation between the chaotic many-body states. Several different ways of defining the temperature and entropy for such systems have been considered in [9-12,14]. Both the sd shell model and the two-body random interaction model showed that for sufficient two-body interaction strengths the occupation numbers agree with the Fermi-Dirac distribution (FDD). This could be expected from the point of view of Landau-Migdal Fermi-liquid theory which describes the excitations of the system in terms of interacting quasiparticles.

On the other hand, our study of Ce [16] demonstrates that there could be serious deviations from the FDD due to strong fluctuations of the two-body interaction between different orbitals, Fig. 6. Nevertheless, it is possible to introduce the temperature, and even describe the non-trivial behaviour of the occupation numbers observed in the configuration-interaction calculations using a thermodynamic approach. The study of the occupation numbers provides a clear illustration of the relation between the statistical equilibrium due to many-body chaos and the usual one, due to interaction of the system with a heat bath.

The occupation number of a given single particle state $\alpha$ in a many-body eigenstate $i$ is given by $\left\langle\Psi_{i}\left|\hat{n}_{\alpha}\right| \Psi_{i}\right\rangle \equiv\left\langle\Psi_{i}\left|a_{\alpha}^{\dagger} a_{\alpha}\right| \Psi_{i}\right\rangle$. It is more instructive to

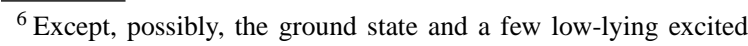
states.
}

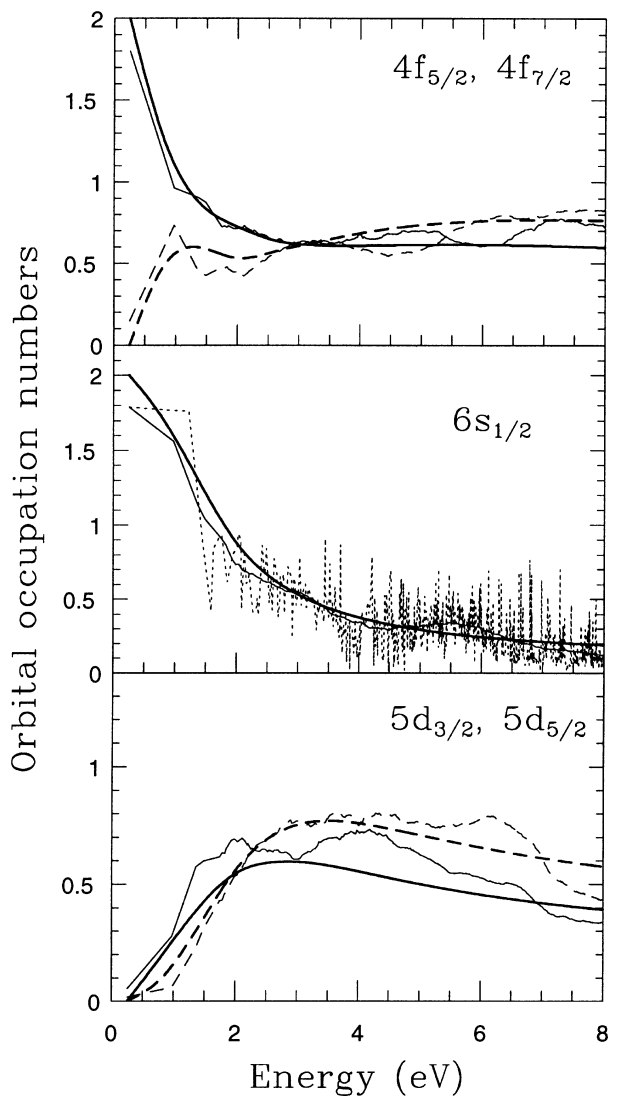

Fig. 6. Energy dependence of the occupation numbers. Thin solid and dashed line show $\overline{\left\langle\Psi_{i}\left|\hat{n}_{\alpha}\right| \Psi_{i}\right\rangle}$ calculated numerically and averaged over an energy window. Thick solid and dashed lines are the result of the thermodynamic description equations (11) and (12). The solid and dashed lines correspond to the lower and upper fine-structure components $\left(4 f_{5 / 2}\right.$ and $4 f_{7 / 2}$, and $5 d_{3 / 2}$ and $5 \mathrm{~d}_{5 / 2}$, respectively). Dotted line in the middle plate connects $n_{6 s}$ calculated for every eigenstate.

average these occupation numbers over a few neighbouring eigenstates with $E^{(i)} \approx E$ and define $n_{\alpha}(E)$ as

$$
\begin{aligned}
n_{\alpha}(E)= & \overline{\left\langle\Psi_{i}\left|\hat{n}_{\alpha}\right| \Psi_{i}\right\rangle}=\sum_{k}\left\langle C_{k}^{2}\right\rangle_{E}\left\langle n_{\alpha}\right\rangle_{k} \\
& \approx \int \rho_{\mathrm{W}}(E, k)\left\langle n_{\alpha}\right\rangle_{k} \mathrm{~d} E_{k},
\end{aligned}
$$

where $\left\langle n_{\alpha}\right\rangle_{k}=\left\langle\Phi_{k}\left|n_{\alpha}\right| \Phi_{k}\right\rangle$, and the summation over $k$ has been replaced by integration using Eq. (6). We also use the similarity between the eigenvalue density $\rho(E)$ and that of the basis state energies $E_{k}$. 


\subsection{Temperature}

Let us now suppose the off-diagonal part of the residual interaction (2) is switched off. The basis states $\left|\Phi_{k}\right\rangle$ then represent the eigenstates of the system with energies

$$
\begin{aligned}
E_{k}= & \sum_{\alpha} \varepsilon_{\alpha}\left\langle n_{\alpha}\right\rangle_{k} \\
& +\sum_{\alpha<\beta}\left(V_{\alpha \beta \beta \alpha}-V_{\alpha \beta \alpha \beta}\right)\left\langle n_{\alpha}\right\rangle_{k}\left\langle n_{\beta}\right\rangle_{k} .
\end{aligned}
$$

If we bring the system in contact with a heat bath at temperature $T$ the probabilities of finding the system in different states $k$ will be given by the canonical distribution $w_{k}=Z^{-1} \exp \left(-E_{k} / T\right)$, where $Z=$ $\sum_{k} \exp \left(-E_{k} / T\right)$. Accordingly, the average occupation number at temperature $T$ is given by

$n_{\alpha}(T)=\sum_{k} w_{k}\left\langle n_{\alpha}\right\rangle_{k} \simeq \int w_{T}\left(E_{k}\right)\left\langle n_{\alpha}\right\rangle_{k} \mathrm{~d} E_{k}$

where $w_{T}\left(E_{k}\right)=Z^{-1} \exp \left(-E_{k} / T\right) \rho\left(E_{k}\right)$ is the canonical probability density. It peaks at some energy because of the competition between the two exponents (see Eq. (4)). The position of the peak gives the usual thermodynamic relation between the most probable energy of the system and the temperature, $T^{-1}=\mathrm{d}[\ln \rho(E)] / \mathrm{d} E$. If the temperature is not too small the energy defined by this equation is close to the mean energy

$E(T)=\sum_{k} w_{k} E_{k} \simeq \int E_{k} w_{T}\left(E_{k}\right) \mathrm{d} E_{k}$.

Compare Eqs. (9) and (11). In both the cases the occupation numbers of the basis states are folded with a bell-shaped distribution, provided either by the strength function (spreading due to the residual interaction which mixes the basis states), or by the thermodynamic probability density ("mixing" due to interaction with a heat bath). In this sense the mixing produced by the strong two-body interaction can be indeed viewed as an equilibrium similar to the thermodynamic one. Besides, one can use the statistical model given by Eqs. (10)-(12) to find the relation between the energy and temperature ${ }^{7}$ and calculate the occupation numbers in the system of few strongly interacting particles.

Using the single-particle energies and the two-body matrix elements for the 14 lowest electron orbitals in Ce we have performed the thermodynamic calculation of the occupation numbers for the even states of $\mathrm{Ce}$. For a rotationally symmetric system it is more convenient to look at the occupation numbers of the orbitals $n l j: \hat{n}_{n l j}=\sum_{j_{z}} a_{n l j j_{z}}^{\dagger} a_{n l j j_{z}}$, which are independent of the $z$ components $j_{z}$ of the angular momentum, as well as of $J_{z}$ (see [16] for details). The results are shown in Fig. 6 by thick curves. They fit well the energy dependence of the occupation numbers from the exact Hamiltonian diagonalization. For the 6 s orbital we also show the unaveraged occupation numbers. At $E>2 \mathrm{eV}$ the fluctuations of $n_{6 \mathrm{~s}}$ become small. This boundary corresponds to the onset of strong mixing and chaotic eigenstates in the system.

\section{Matrix elements between chaotic eigenstates}

\subsection{Statistical theory}

The random-like behaviour of the components of the chaotic many-body eigenstates (Fig. 2) means that the matrix elements of an operator $\hat{M}$ calculated for such states

$M_{a b}=\left\langle\Psi_{a}|\hat{M}| \Psi_{b}\right\rangle=\sum_{k, l} C_{k}^{(a)} C_{l}^{(b)}\left\langle\Phi_{k}|\hat{M}| \Phi_{l}\right\rangle$

display Gaussian statistics, as any sum of a large number of uncorrelated random variables. This results in the Porter-Thomas statistics of the probabilities proportional to the square of (13) - a fact well known for nuclear processes involving compound (chaotic) states [5]. In complex many-body systems there is no hope of calculating the highly excited chaotic eigenstates

\footnotetext{
${ }^{7}$ The true relation between $E$ and $T$ should include a correction, $E=E(T)-\Delta_{E}(T)$, where $\Delta_{E}(T)$ is positive in the lower (physical) part of the spectrum. It takes into account the shift of the eigenvalues with respect to the basis states due to the offdiagonal part of the Hamiltonian [14-16]. In fact, $T>0$ even in the ground states of the system, since there is admixture of some higher lying configuration to it.
} 
accurately enough to allow state-by-state comparison with the experiment. The same is true for the transition amplitudes (13) between them. In some cases the spectra can be so dense that it becomes impossible to resolve particular levels, and the experiment can only produce some average characteristics. Thus, it is important to be able to calculate the mean-squared values of the transition amplitudes. Averaged over levelto-level fluctuations they will vary smoothly with the energies of the states involved.

A statistical approach to calculation of meansquared values of the matrix elements between chaotic many-body states has been developed and described in $[8,17,18,32]$, and we will only present the results here. Suppose $\hat{M}$ is a one-body operator ${ }^{8} \hat{M}=$ $\sum_{\alpha, \beta}\langle\alpha|\hat{m}| \beta\rangle a_{\alpha}^{\dagger} a_{\beta} \equiv \sum_{\alpha, \beta} m_{\alpha \beta} \hat{\rho}_{\alpha \beta}$, which causes transitions between the chaotic states $a$ and $b$. Eq. (13) then becomes

$\left\langle\Psi_{a}|\hat{M}| \Psi_{b}\right\rangle=\sum_{\alpha, \beta} m_{\alpha \beta} \rho_{\alpha \beta}^{(a b)}$,

where $\rho_{\alpha \beta}^{(a b)}=\left\langle\Psi_{a}\left|a_{\alpha}^{\dagger} a_{\beta}\right| \Psi_{b}\right\rangle$ is a nondiagonal matrix element of the density matrix operator. It gives the contribution of the single-particle transition $\alpha \rightarrow \beta$ in the many-body matrix elements between the states $a$ and $b$. The mean value of the matrix element (13) is zero if $a \neq b$ (in many applications states $a$ and $b$ are even of different symmetry, e.g., if one examines electromagnetic $E 1$ transitions they must have opposite parities). For chaotic many-body eigenstates contributions of transitions between different pairs of singleparticle states $\alpha, \beta$ are uncorrelated, and the meansquared matrix element is given by

$\overline{\left|\left\langle\Psi_{a}|\hat{M}| \Psi_{b}\right\rangle\right|^{2}}=\sum_{\alpha, \beta}\left|m_{\alpha \beta}\right|^{2} \overline{\left|\rho_{\alpha \beta}^{(a b)}\right|^{2}}$.

The main result of the statistical theory is that the mean-squared value of the density matrix operator can be expressed in terms of the parameters of the chaotic eigenstates $a$ and $b$ and the average occupation num-

\footnotetext{
${ }^{8}$ For example, the interaction with the electromagnetic field, or the parity-violating weak potential. One can also consider matrix elements of two-body operators $[17,18]$.
}

bers of the single-particle states $\alpha$ and $\beta$ in the following two forms:

$$
\begin{aligned}
& \overline{\left|\rho_{\alpha \beta}^{(a b)}\right|^{2}}=D_{a} \tilde{\delta}\left(\Gamma_{a}, \Gamma_{b}, \Delta\right)\left\langle\hat{n}_{\beta}\left(1-\hat{n}_{\alpha}\right)\right\rangle_{b}, \\
& \overline{\left|\rho_{\alpha \beta}^{(a b)}\right|^{2}}=D_{b} \tilde{\delta}\left(\Gamma_{a}, \Gamma_{b}, \Delta\right)\left\langle\hat{n}_{\alpha}\left(1-\hat{n}_{\beta}\right)\right\rangle_{a},
\end{aligned}
$$

where $D_{a, b}$ are the mean level spacings for the states $a$ and $b$, and $\tilde{\delta}$ is a "finite-width $\delta$ function". It depends on the spreading widths $\Gamma_{a, b}$ of the eigenstates and on the energy difference $\Delta=\omega_{\beta \alpha}-E^{(b)}+E^{(a)}$ between the many-body state energies $E$ and the energy of the single-particle transition $\omega_{\beta \alpha}=\varepsilon_{\beta}-\varepsilon_{\alpha}$. The exact form of the function $\tilde{\delta}\left(\Gamma_{a}, \Gamma_{b}, \Delta\right)$ depends on the spreading of the many-body states over the basis components $\left\langle C_{\underset{\kappa}{k}}^{2}\right\rangle_{E}$. If its shape is described by the BW equation (7), $\tilde{\delta}$ also has a Breit-Wigner profile

$\tilde{\delta}\left(\Gamma_{a}, \Gamma_{b}, \Delta\right)=\frac{1}{2 \pi} \frac{\Gamma_{a}+\Gamma_{b}}{\Delta^{2}+\left(\Gamma_{a}+\Gamma_{b}\right)^{2} / 4}$.

It describes the specific "energy conservation" in transitions between the chaotic multicomponent eigenstates and has a maximum at $E^{(b)}-E^{(a)}=\varepsilon_{\beta}-\varepsilon_{\alpha}$.

In systems with rotationally symmetric Hamiltonians the eigenstates are characterized by the $J$ and $J_{z}$ values. Thanks to the Wigner-Ekhart theorem one only needs to calculate the reduced matrix element $\left\langle\Psi_{a}\|\hat{M}\| \Psi_{b}\right\rangle$ independent of the $J_{z}$ values of the states $a$ and $b$. At the same time, one should replace $m_{\alpha \beta}$ with the reduced single-particle amplitudes and use occupation numbers of the orbitals $n l j$ rather than the single-particle states $\alpha \equiv n l j j_{z}$ (cf. Section 3.2). The expression for the mean-squared reduced matrix element retains the structure of Eqs. (15) and (16), but must be modified (see $[8,32]$ ). The result will depend on the rank $k$ of the irreducible spherical tensor operator $\hat{M}$. For $J_{a}=J_{b}$ the mean-squared reduced matrix element can be estimated as

$$
\begin{aligned}
& \overline{\left|\left\langle\Psi_{a}\|\hat{M}\| \Psi_{b}\right\rangle\right|^{2}} \\
& \quad \approx \frac{2 J_{a}+1}{2 k+1} D_{a} \sum_{n l j, n^{\prime} l^{\prime} j^{\prime}}\left|\left\langle n l j\|\hat{m}\| n^{\prime} l^{\prime} j^{\prime}\right\rangle\right|^{2} \\
& \quad \times \tilde{\delta}\left(\Gamma_{a}, \Gamma_{b}, \Delta\right)\left\langle\frac{n_{n^{\prime} l^{\prime} j^{\prime}}}{2 j^{\prime}+1}\left[1-\frac{n_{n l j}}{2 j+1}\right]\right\rangle_{b},
\end{aligned}
$$

or a similar form corresponding to the lower expression in Eq. (16). 


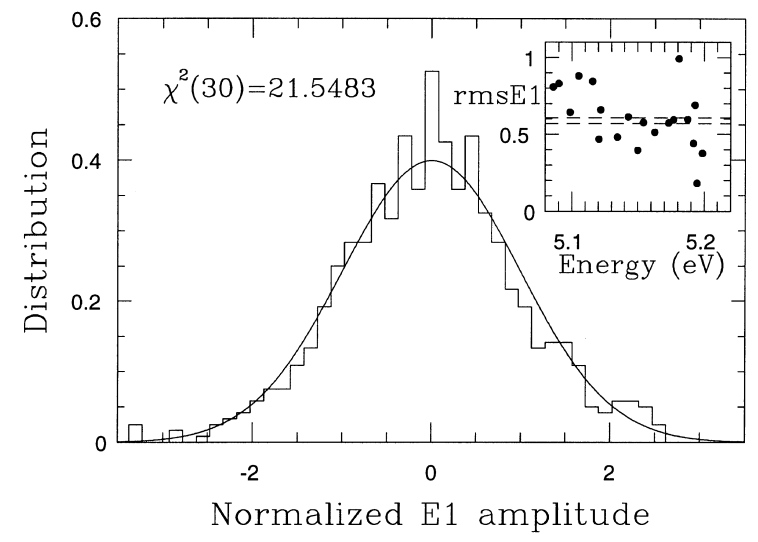

Fig. 7. Statistics of the normalized $E 1$ amplitudes $M_{a b}^{(n)}$ for transitions between the 191-210 odd and 261-280 even states with $J=4$ in Ce (histogram). The solid line is the normal Gaussian distribution. The inset shows the rms values $\left[\left\langle M^{2}\right\rangle_{a}\right]^{-1 / 2}$ of the $E 1$ amplitude (in units of the Bohr radius $a_{0}$ ). Dashed lines are the values obtained from the statistical theory, Section 4.1.

\subsection{Numerical results}

We illustrate the theory outlined above by studying the amplitudes $M_{a b}$ of electromagnetic dipole $(E 1)$ transitions between the 191-210 odd and 261-280 even states with $J=4$ in Ce. These states lie around $5 \mathrm{eV}$ above the ground state, far into the quantum chaotic region ${ }^{9}$. To separate out level-to-level fluctuations of the amplitude from the slow secular variation of its rms value we normalize the amplitudes as $M_{a b}^{(n)}=M_{a b}\left[\left\langle M^{2}\right\rangle_{a}\right]^{-1 / 2}$, where $\left\langle M^{2}\right\rangle_{a}$ is calculated for all odd states $a$ by averaging $M_{a b}^{2}$ over the 40 even states. The statistics obtained for the 800 amplitudes $M_{a b}^{(n)}$ is shown on Fig. 7. It agrees well with the Gaussian distribution with zero mean and unit variance.

The Gaussian statistics of the amplitude is a trivial consequence of the chaotic nature of the eigenstates involved. It is much more important to see how the statistical theory of Section 4.1 works for its meansquared value. The inset in Fig. 7 shows the rms amplitudes $\left[\left\langle M^{2}\right\rangle_{a}\right]^{-1 / 2}$ as functions of the energy of the odd states $E_{a}$. To check the theory we first determine

\footnotetext{
${ }^{9}$ In [32] we looked at the transitions between the 14 lowest $J^{\pi}=4^{-}$states below the quantum chaos boundary and the 20$100 J^{\pi}=4^{-}$states, which are already chaotic. It is in fact sufficient if only one of the states $a$ or $b$ is chaotic to make the consideration of Section 4.1 valid.
}

the parameters $N$ and $\Gamma$ and $D$ of the odd and even eigenstates involved. This is done by fitting BW profiles to $\left\langle C_{k}^{2}\right\rangle_{E}$ for them, as shown in Fig. 2(b). We can also find the average occupation numbers of the orbitals involved. Both procedures are done for the eigenstates in the middle of the energy intervals studied: $200 \pm 9$ (odd) and $260 \pm 9$ (even). The result is then obtained from Eq. (18). The two forms of the answer (cf. Eq. (16)) yield slightly different values (0.607 and 0.569) shown in Fig. 7 (inset) with dashed lines. They are close to the numerical rms value of 0.637 obtained as an average over all 800 transitions.

Of course, the main goal of our statistical theory is not to reproduce the results obtained by exact diagonalization of the Hamiltonian matrix. There are many complex systems (e.g., compound nuclei) where the size of the Hilbert space makes exact diagonalization impossible. Nevertheless, the statistical theory should enable one to estimate all important parameters of the system, such as the density of states, spreading widths of the chaotic many-body eigenstates, orbital occupation numbers, and finally, the mean-squared transition amplitudes between the chaotic states. From this point of view $\mathrm{Ce}$ is nothing but a convenient testing ground. The main ideas and approaches should be applicable with some little modifications to the whole variety of finite Fermi systems with strong interaction between particles.

\section{Dynamical enhancement of perturbations}

Consider a many-body quantum chaotic system whose properties are similar to those studied above for the Ce atom. Suppose a weak perturbation $\hat{M}$ that mixes states of different symmetry is applied. This can be an external electric field, or the weak interaction between the electrons and the nucleus in an atom, or between the nucleons in a nucleus. The effect produced by the perturbation (e.g., parity violation) is proportional to the mixing coefficient

$\eta_{a b}=\frac{\left\langle\Psi_{a}|\hat{M}| \Psi_{b}\right\rangle}{E_{a}-E_{b}}$.

The strongest mixing takes place between nearby states $\left|E_{a}-E_{b}\right| \sim D$, where $D$ is exponentially small 
compared to typical single-particle spacings $\omega_{\alpha \beta}$. The rms matrix element between the chaotic states estimated in Section 4.1 is, for $E_{a} \approx E_{b}: M_{a b} \sim$ $\left(D m_{\alpha \beta}^{2} q / \Gamma\right)^{1 / 2} \sim m_{\alpha \beta} \sqrt{q / N}$, where $m_{\alpha \beta}$ here is the typical single-particle matrix element, $\Gamma$ is the larger of the two spreading widths, and $q$ is the effective number of single-particle transitions that contribute to the sum in Eq. (15), comparable to the number of active particles. Thus, the matrix element between chaotic states is suppressed as $1 / \sqrt{N}$ with respect to simple single-particle matrix elements. Nevertheless, the typical mixing of the chaotic many-particle states

$\eta \sim M_{a b} / D \sim \sqrt{q N} m_{\alpha \beta} / \Gamma \gg m_{\alpha \beta} / \omega_{\alpha \beta}$

is $\sqrt{N}$ times enhanced compared to the single-particle mixing. The last inequality implies $\Gamma \sim \omega_{\alpha \beta}$, which is usually true. This effect characteristic for manybody systems with strong interaction between particles and dense spectra is often referred to as dynamical enhancement of perturbations ${ }^{10}$.

There is no repulsion between the energy levels of different symmetry, and the distance to the nearest neighbour should be described by the Poisson distribution. If we take into account that the matrix elements $M_{a b}$ obey Gaussian statistics, the mixings $\eta$ will be distributed according to the Cauchy distribution: $f_{c}(\eta)=\left(\eta_{c} / \pi\right) /\left(\eta^{2}+\eta_{c}^{2}\right)$, where $\eta_{c}$ is the ratio of the rms matrix element to the mean level spacing between the mixed levels [41]. At large $\eta f(\eta) \propto \eta^{-2}$, hence the variance of the mixing is infinite, and the mean is zero only in the principal value sense. This means that large mixings are highly probable. The origin of this effect is the absence of repulsion between levels of different symmetries and the large probability of coming across very small $E_{a}-E_{b}$ in Eq. (19). There is another interesting property which follows from the infinite variance. Such random variables do not obey the central limit theorem. Consequently, the average value of $n$ independent mixings fluctuates as strongly as any single mixing coefficient, and there is no $1 / \sqrt{n}$

\footnotetext{
${ }^{10}$ In principle, this effect takes place for perturbations which mix states of the same symmetry as well. For example, in complex open-shell atoms it aids the removal of the conservation of the total spin $S$ and orbital angular momentum $L$ by the spin-orbit interaction.
}

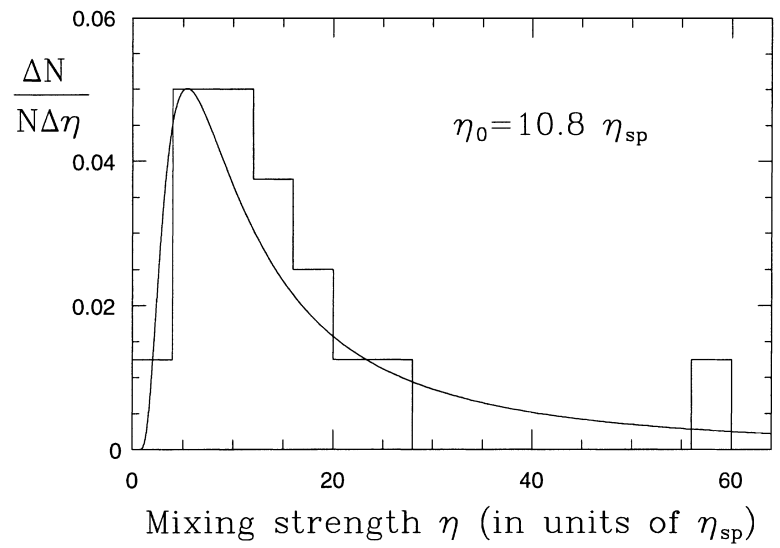

Fig. 8. Statistics of the maximal mixings for $20 \mathrm{~J}^{\pi}=4^{-}$levels in Ce (191-210) with even $J=4$ states, by the electric field $E 1$ transition operator. Solid curve is Eq. (21) fitted to the numerical data.

suppression. Therefore, averaging over $n$ consecutive levels (e.g., due to poor experimental resolution) does not suppress the dynamical enhancement effect [41].

If instead of nearest-neighbour mixing we consider the maximal absolute value of the mixing for a given state their distribution can be approximated by

$f(\eta)=\left(\eta_{0} / \eta^{2}\right) \exp \left(-\eta_{0} / \eta\right)$

where $\eta_{0} \sim \eta_{c}$ is the characteristic value of the maximal mixing [8]. Both the mean and the variance are infinite for this distribution. Fig. 8 illustrates the distribution of maximal mixings and the existence of dynamical enhancement in Ce. To make the latter more obvious we present $\eta$ values in units of $\eta_{\mathrm{sp}}$ - the typical single-particle mixing. We estimated it from the $7 \mathrm{~s}-7 \mathrm{p}$ transition in Ce: $\eta_{\mathrm{sp}}=\langle 7 \mathrm{p}\|E 1\| 7 \mathrm{~s}\rangle /\left(\varepsilon_{7 \mathrm{p}}-\varepsilon_{7 \mathrm{~s}}\right) \approx 500$ a.u. ${ }^{11}$. Note that for the Cs atom $(Z=55)$ which has only one valence electron and a very simple spectrum $\eta_{7 \mathrm{~s} 7 \mathrm{p}} \approx 800$ a.u., close to our estimate of $\eta_{\mathrm{sp}}$ in Ce.

The value of $\eta_{0}=10.8 \eta_{\mathrm{sp}}$ obtained from the fit characterizes the magnitude of the enhancement factor. This value could also be estimated by dividing the rms $E 1$ amplitude for these states, $0.637 a_{0}$, by the mean distance $D / 2=2.7 \mathrm{meV}$ from the odd state to the nearest $J^{\pi}=4^{+}$state in this part of the spectrum: $\eta_{0} \sim 13 \eta_{\text {sp. }}$. Note that there are much large mixings

\footnotetext{
${ }^{11}$ For the mixing by an electric field 1 atomic unit equals $3.67 \times$ $10^{-2} a_{0} \mathrm{eV}^{-1}$.
} 
in Fig. 8. For the three out of 20 levels the maximal mixings fall outside the plot: $\eta=137,244$ and $568 \eta_{\mathrm{sp}}$ (manifestation of the slow drop of $f(\eta)$ ). Thus, the dynamical enhancement increases the mixing of states by the external perturbation in Ce by a factor of more than 10. Note that for the eigenstates considered the number of principal components is $N \sim 300$, and the $\sqrt{N}$ estimate of the enhancement is confirmed. In more complicated systems the enhancement can be much stronger, e.g., in heavy nuclei where $N \sim 10^{6}$, the dynamical enhancement factor reaches $10^{3}$.

Some of the properties of many-body quantum chaotic systems studied in this work depend on the choice of the single-particle and many-body basis sets. The natural choice for them is provided by the mean field of the system. However, the mean field cannot be defined uniquely for open-shell systems. To this extent the basis-dependent properties of the system, e.g., the number of principal components or the single-particle occupation numbers may vary with the change of the basis. Unlike them, the effect of dynamical enhancement is basis independent. It can be directly observed and measured, and its magnitude is an important characteristic of the "degree" of quantum chaos in the system.

\section{Summary}

We have presented the results of an extensive case study of quantum chaos in a realistic many-body system - the atom of cerium. The properties of its eigenvalue spectra are quite generic for such systems, and so should be those of the chaotic many-body eigenstates. The eigenstates are characterized by the spreading of their basis components, which is described well by the Breit-Wigner profile around its maximum. Together with the level density the spreading width is one of the most important characteristics of the eigenstates.

We have shown how to develop a statistical theory of these systems based on the structure of the eigenstates, and compared its results with direct numerical calculations in $\mathrm{Ce}$. The theory enables one to introduce temperature and calculate the occupation numbers using a thermodynamic approach. These occupa- tion numbers and the parameters of the chaotic eigenstates are needed to obtain mean-squared values of transition amplitudes due to an external perturbation from the statistical theory. Using $\mathrm{Ce}$ as an example we have demonstrated the existence of dynamical enhancement of perturbations in many-body chaotic systems. This effect may be viewed as a quantum counterpart of the exponential divergence of trajectories (high sensitivity to the initial conditions in classically chaotic systems). It can also be important for the problem of quantum measurement.

Our investigation shows that heavy atoms are natural testing grounds for studying many-body quantum chaos. Quantum chaotic regime can possibly be achieved in any atom by exciting a sufficient number of electrons. Actinides and especially positive atomic ions [42] can be among the most interesting objects for future studies.

\section{Acknowledgements}

We would like to thank M.G. Kozlov and F.M. Izrailev who participated in some of the work reviewed here, and B.V. Chirikov for his keen interest in our work on chaos in the $\mathrm{Ce}$ atom. This research has been supported by the Australian Research Council.

\section{References}

[1] D.L. Shepelyansky, O.P. Sushkov, Europhys. Lett. 37 (1997) 121.

[2] B.L. Altshuler, Yu. Gefen, A. Kamenev, S.L. Levitov, Phys. Rev. Lett. 78 (1997) 2803.

[3] A.D. Mirlin, Y.V. Fyodorov, Phys. Rev. B 56 (1997) 13393.

[4] P. Jaquod, D.L. Shepelyansky, Phys. Rev. Lett. 79 (1997) 1837.

[5] A. Bohr, B. Mottelson, Nuclear Structure, vol. 1, Benjamin, New York, 1969.

[6] N. Rosenzweig, C.E. Porter, Phys. Rev. 120 (1960) 1698.

[7] H.S. Camarda, P.D. Georgopulos, Phys. Rev. Lett. 50 (1983) 492.

[8] V.V. Flambaum, A.A. Gribakina, G.F. Gribakin, M.G. Kozlov, Phys. Rev. A 50 (1994) 267.

[9] M. Horoi, V. Zelevinsky, B.A. Brown, Phys. Rev. Lett. 74 (1995) 5194

[10] M. Horoi, V. Zelevinsky, B.A. Brown, Phys. Lett. B 350 (1995) 141. 
[11] N. Frazier, B.A. Brown, V. Zelevinsky, Phys. Rev. C 54 (1996) 1665

[12] V. Zelevinsky, B.A. Brown, N. Frazier, M. Horoi, Phys. Rep. 276 (1996) 85.

[13] V.V. Flambaum, F.M. Izrailev, G. Casati, Phys. Rev. E 54 (1996) 2136.

[14] V.V. Flambaum, F.M. Izrailev, Phys. Rev. E 55 (1997) R13.

[15] V.V. Flambaum, F.M. Izrailev, Phys. Rev. E 56 (1997) 5144.

[16] V.V. Flambaum, A.G. Gribakina, G.F. Gribakin, I.V. Ponomarev, Phys. Rev. E 57 (1998) 4933.

[17] V.V. Flambaum, O.K. Vorov, Phys. Rev. Lett. 70 (1993) 4051.

[18] V.V. Flambaum, in: C.R. Gould, J.D. Bowman, Yu.P. Popov (Eds.), Time Reversal and Parity Violation in Neutron Reactions, World Scientific, Singapore, 1994, pp. 39-45.

[19] O.P. Sushkov, V.V. Flambaum, Usp. Fiz. Nauk 136 (1982) 3 [Sov. Phys. Usp. 25 (1982) 1].

[20] V.V. Flambaum, G.F. Gribakin, Prog. Part. Nucl. Phys. 35 (1995) 423.

[21] T.A. Brody, J. Flores, J.B. French, P.A. Mello, A. Pandey, S.S.M. Wong, Rev. Mod. Phys. 53 (1981) 385.

[22] T. Guhr, A. Müller-Groeling, H.A. Weidenmüller, Phys. Rep. (1998), cond-mat/9707301, to be published.

[23] G. Casati, B.V. Chirikov, Quantum Chaos: Between Order and Disorder, Part 2, Cambridge University Press, Cambridge, 1995.

[24] M. Courtney, D. Kleppner, Phys. Rev. A 53 (1996) 178, and references therein.

[25] D. Delande, J.C. Gay, Phys. Rev. Lett. 57 (1986) 2006.

[26] M.V. Berry, Ann. Phys. 131 (1981) 163.
[27] O. Bohigas, M.J. Giannoni, C. Schmit, Phys. Rev. Lett. 52 (1983) 1

[28] B.V. Chirikov, Phys. Lett. A 108 (1985) 68.

[29] W.C. Martin, R. Zalubas, L. Hagan, Atomic Energy Levels - The Rare-Earth Elements, Natl. Bur. Stand. Ref. Data Ser. Natl. Bur. Stand. (US), NBS-60, (US, GPO, Washington, DC, 1978).

[30] A.A. Gribakina, V.V. Flambaum, G.F. Gribakin, Phys. Rev. E 52 (1995) 5667.

[31] V.V. Flambaum, A.A. Gribakina, G.F. Gribakin, Phys. Rev. A 54 (1996) 2066

[32] V.V. Flambaum, A.A. Gribakina, G.F. Gribakin, Phys. Rev. A 58 (1998) 230.

[33] J.P. Connerade, M.A. Baig, M. Sweeney, J. Phys. B 23 (1990) 713.

[34] J.P. Connerade, I.P. Grant, P. Marketos, J. Oberdisse, J. Phys. B 23 (1995) 2539.

[35] J.P. Connerade, J. Phys. B 30 (1997) L31.

[36] Th. Zimmermann, H. Köppel, L.S. Cederbaum, Phys. Rev. Lett. 61 (1988) 3.

[37] E.P. Wigner, Ann. Math. 62 (1955) 548.

[38] E.P. Wigner, Ann. Math. 65 (1957) 203.

[39] Y.V. Fyodorov, O.A. Chubykalo, F.M. Izrailev, G. Casati, Phys. Rev. Lett. 76 (1996) 1603.

[40] B. Georgeot, D.L. Shepelyansky, Phys. Rev. Lett. 79 (1997) 4365.

[41] V.V. Flambaum, G.F. Gribakin, Phys. Rev. C 50 (1994) 3122.

[42] G.F. Gribakin, A.A. Gribakina, V.V. Flambaum, Aust. J. Phys. 52 (1999) No. 3. 